\title{
Contributions of Nitric Oxide Synthases, Dietary Nitrite/Nitrate, and Other Sources to the Formation of NO Signaling Products
}

\author{
Alexandra B. Milsom, Bernadette O. Fernandez,,2 Maria F. Garcia-Saura, \\ Juan Rodriguez, ${ }^{3 *}$ and Martin Feelisch ${ }^{1,2^{*}}$
}

\begin{abstract}
Mice lacking all three nitric oxide synthase (NOS) genes remain viable even though deletion of the major downstream target of $\mathrm{NO}$, soluble guanylyl cyclase, is associated with a dramatically shortened life expectancy. Moreover, findings of relatively normal flow responses in eNOS knockouts are generally attributed to compensatory mechanisms including upregulation of remaining NOS isoforms, but the alternative possibility that dietary nitrite/nitrate (NOx) may contribute to basal levels of NO signaling has never been investigated. Aim: The aim of the present study was to examine how NO signaling products (nitrosated and nitrosylated proteins) and NO metabolites (nitrite, nitrate) are affected by single NOS deletions and whether dietary NOx plays a compensatory role in any deficiency. Specifically, we sought to ascertain whether profound alterations of these products arise upon genetic deletion of either NOS isoform, inhibition of all NOS activity, NOx restriction, or all of the above. Results: Our results indicate that while some significant changes do indeed occur, they are surprisingly moderate and compartmentalized to specific tissues. Unexpectedly, even after pharmacological inhibition of all NOSs and restriction of dietary NOx intake in eNOS knockout mice significant levels of NOrelated products remain. Innovation/Conclusion: These findings suggest that a yet unidentified source of NO, unrelated to NOSs or dietary NOx, may be sustaining basal NO signaling in tissues. Given the significance of NO for redox regulation in health and disease, it would seem to be important to identify the nature of this additional source of NO products as it may offer new therapeutic avenues for correcting NO deficiencies. Antioxid. Redox Signal. 17, 422-432.
\end{abstract}

\section{Introduction}

$\mathbf{N}$ ITRIC OXIDE (NO) IS A CRITICAL MOLECULE in numerous cell signaling pathways that is essential to maintaining health, including host defense, neuronal communication, and the control of vascular tone (22); in addition, it is involved in pathophysiological mechanisms (13). Its production from Larginine is controlled by three distinct isoforms of NO synthase (NOS), coded for by three distinct genes with discrete cell/tissue localization, catalytic properties, regulation, and inhibitor sensitivities (24). More recently, the role of a fourth NO signaling source, nitrite, has become increasingly apparent $(3,18)$. This source is of particularly interest due to its chemical stability in vivo and because it can be derived, either directly or via reduction of nitrate, from a normal diet. In addition to locally produced NO itself, a circulating pool of longer-lived NO metabolites, including nitrite, nitrate, Snitroso and $\mathrm{N}$-nitroso species (27), can give rise to $\mathrm{NO}$ following reductive bioactivation and cooperates with tissuebound storage forms of $\mathrm{NO}$ undergoing redox-activation $(2,6$, 29) to contribute to overall NO availability. Although there is ample evidence for cross-talk between these NO species, a unifying concept of regulation and the factors that govern their concentrations in different biological compartments is lacking. The current view of how these different sources of $\mathrm{NO}$ are related to NO signaling is represented diagrammatically in Figure 1.

The two principal pathways through which NO signals include the binding of NO to heme-containing proteins (chemically, a nitrosylation reaction), exemplified by activation of the enzyme soluble guanylyl cyclase with production of the second messenger cyclic GMP, and the nitrosation of

${ }^{1}$ Whitaker Cardiovascular Institute, Boston University School of Medicine, Boston, Massachusetts.

${ }^{2}$ Division of Metabolic \& Vascular Health, University of Warwick Medical School, Coventry, United Kingdom.

${ }^{3}$ Department of Physics, Centenary College of Louisiana, Shreveport, Louisiana.

*These authors contributed equally to this article. 


\section{Innovation}

Adequate production of the ubiquitous signaling and effector molecule nitric oxide (NO) is essential to mammalian health; it is also involved in pathophysiological processes. Three distinct nitric oxide synthases (NOS) have been identified to account for the majority of NO-related cell signaling, and dietary nitrite/nitrate intake and redox cycling of the circulating NO metabolite pool are the only other sources known to contribute to bodily $\mathrm{NO}$ availability. Using established NOS knockout models in combination with pharmacological and dietary manipulation, we here provide evidence for the existence of one or more unrecognized source(s) of NO production in vivo. Our results suggest that this unidentified source may be linked to mitochondrial activity. Given the significance of NO for redox regulation in health and disease, it would seem to be important to identify the nature of this additional source of NO production and characterize its role and significance for cell regulation and redox homeostasis. Moreover, perturbation of $\mathrm{NO}$ formation via this new pathway may also be linked to disease; thus, its identification could offer new therapeutic avenues for correction of any deficiencies that are distinct from the current use of classical NO donors. Our results may be of importance for redox signaling and cell regulation as well as translational research.

critical sulfhydryl groups in proteins, a reaction coined "Snitrosylation". The former has long been thought to mediate the majority of NO's effects in physiology (22), while the latter is believed to represent the prototypic redox-based signaling mechanism in biology (31). In fact, $\mathrm{NO}$ can be considered as a redox array of species (13), including nitrosonium $\left(\mathrm{NO}^{+}\right)$ equivalents and nitroxyl anions $\left(\mathrm{NO}^{-}\right)$in addition to the free radical, each with a distinct biological chemistry $(21,32)$. NO is also a potent antioxidant (36) as well as a regulator of cellular redox status (26) and redox-based gene expression.

Many studies to date have sought to investigate the role of NO through NOS gene-deficient (knockout) animals, including single, double, and triple knockout mice (30). In cardiovascular physiology, the production of NO from eNOS is critical not only for the maintenance of vascular tone but also for its antithrombotic, antiproliferative, and anti-inflammatory actions

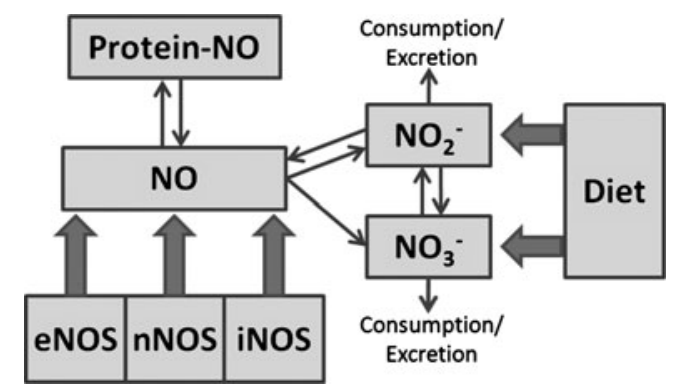

FIG. 1. Schematic representation of the sources contributing to the bodily NO pool. eNOS, endothelial NOS; iNOS, inducible NOS; nNOS, neuronal NOS; NO, bodily pool of nitric oxide related signaling products; $\mathrm{NO}_{2}{ }^{-}$, nitrite; $\mathrm{NO}_{3}{ }^{-}$, nitrate; protein-NO, protein-bound $\mathrm{NO}$ storage forms.
(35). Interestingly, transgenic mice lacking a functional eNOS gene are viable and, although mildly hypertensive, show normal flow responses in various vascular beds. These findings have most commonly been attributed to compensatory pathways, including upregulation of remaining NOS isoforms, soluble guanylate cyclase activity, and endothelial-derived hyperpolarizing factor (12). Of particular interest was the development of triple NOS knockouts (33). The realization that these animals are viable is surprising given that total deletion of the major downstream target of $\mathrm{NO}$, soluble guanylyl cyclase, is known to be associated with dramatically reduced life expectancy (19). Taken together, these findings invite questions on the compensatory role of extra-NOS sources in maintaining basal levels of NO signaling that ultimately may help keep total NOS-knockouts viable.

Earlier studies have demonstrated that dietary restriction of nitrite and nitrate (NOx) intake is associated with changes in NO signaling products in a direction opposite to that of an increase in nitrite and/or NO availability (3). Conversely, blood levels of nitrosyl hemoglobin increase when mice are fed a nitrate-rich diet (5). The aim of the current study was to investigate whether compensatory reactions take place in NOS-deficient animals fed a low-NOx diet. Rather than addressing this question from the perspective of physiological or cell signaling responses, as has been followed in the past, our present study focuses on the alterations in NO signaling products (nitrosated, nitrosylated proteins) and NO metabolites (nitrite, nitrate) found in these animals, species known to be associated with $\mathrm{NO}$ bioactivity (and elevated following, for example, genetic eNOS overexpression (8), iNOS upregulation in inflammation (7), nitrite elevation (3), and NO inhalation (23)). Towards that goal our aim was to ascertain whether significant alterations of these products take place upon: i) genetic deletion of each of the three NOS isoforms; ii) inhibition of all NOS activity; iii) restriction of dietary NOx intake; or iv) all of the above. Surprisingly, we find that none of these interventions eliminates NO signaling, leading us to conclude that a new source of NO is at play. This new source of NO signaling products may offer an explanation as to why triple knockout mice remain viable by suggesting the involvement of additional mechanisms to maintain basal $\mathrm{NO}$ levels.

\section{Results}

\section{Effect of eNOS, nNOS, and iNOS gene deletion on protein-bound NO and NOx levels}

Basal levels of protein-bound NO products found in nNOS, iNOS, and eNOS knockout mice, here collectively expressed as "total nitrosylation" products", are shown in Figure 2 (left panels). Individual steady-state concentrations of Snitrosothiol, N-nitrosamines and NO-heme concentrations in tissues can be found in Supplementary Figure 1 online (supplementary data are available online at www.liebertonline .com/ars). Compared to WT, total nitroso and nitrosyl levels changed little with specific removal of a single NOS isoform, with the exception of $\mathrm{nNOS}^{-/-}$animals displaying lower brain nitros(yl)ation products $(p<0.05)$. If one calculates global plasma and tissue NO metabolite concentrations, adjusted for wet weight (see Fig. 2, right panels), total body protein-NO levels do not change significantly following deletion of any of the NOS isoforms. 

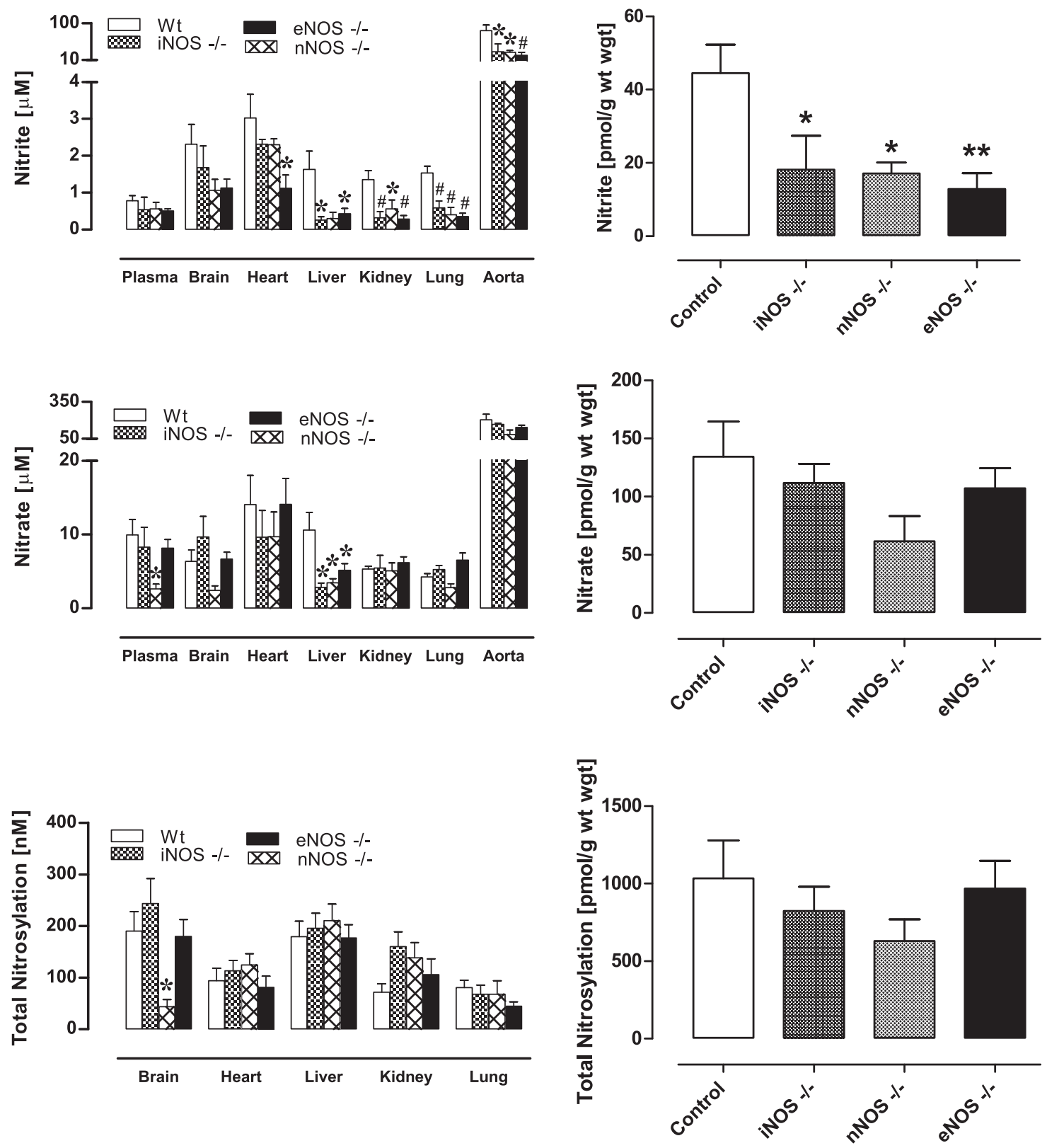

FIG. 2. Contribution of different NOS isoforms to basal NO metabolite concentrations in plasma and tissues of mice. All animals were maintained on a standard rodent chow; wild-type controls (C57BL/6J) are represented by white bars $(n=6-$ 8 ) and isoform-specific knockouts by shaded bars $(n=4-9)$. For illustrative purposes, all protein-bound NO moieties (including S- and N-nitroso and NO-heme species) are displayed as total nitrosylation products (the higher-resolution picture detailing individual changes in either NO species can be found in Supplementary Fig. 1 online). Left-hand panels show steady-state concentrations in individual compartments, right-hand panels display a theoretical total body concentration for each NO metabolite pool as a weighted average. Data are mean \pm SEM, ${ }^{*} p<0.05,{ }^{* *}$ or ${ }^{\#} p<0.01$.

Basal nitrate, the largest contributor to the NO metabolite pool, showed essentially no alteration in steady-state concentration with the removal of individual NOS isoforms. Two exceptions to this general finding were liver nitrate, which was significantly reduced in all NOS knockouts compared with the control group $(p<0.05)$, and a significant reduction in plasma and brain nitrate levels in nNOS-deficient animals $(p<0.05)$. However, none of the NOS genetic deletions had a significant effect on total body nitrate.

In terms of impact on nitrite, the genetic deletion of either nNOS, iNOS, or eNOS resulted in a significant reduction in plasma and tissue nitrite levels as compared to WT controls (Fig. 2, top panels). The reduction is particularly evident in the graphs depicting whole body levels of nitrite, with a highly significant reduction seen in eNOS knockouts.

\section{Effect of low NOx diet on protein-bound NO and NOx levels}

To investigate the contribution of dietary NOx to the steady-state concentrations of $\mathrm{NO}$ metabolites across bodily compartments, WT animals were placed on a low-NOx diet 
for 7 days prior to the analysis of tissues and blood. The results obtained after restriction of dietary NOx intake are shown in Figure 3. Compared to a normal diet, animals placed on a low-NOx diet showed a trend towards reduction, but no statistically significant changes in protein-NO or NOx levels, in plasma or in either of the tissue compartments studied. Although red blood cells were not included in our studies in mice, these results are consistent with data from another investigation demonstrating that changes in nitrosyl hemoglobin levels in the blood of WT mice fed a low-nitrate diet are rather modest when compared to a normal diet (5).

\section{Combined effect of NOS gene deletion or NOS inhibition, and low NOx diet, on protein-bound NO and NOx levels}

To study the combined effect of dietary NOx restriction and NOS inhibition, we investigated a further subset of WT animals on a low NOx diet additionally receiving the pan-NOS inhibitor L-NIO to block endogenous NO production. The results of these experiments are also shown in Figure 3. Total body nitrite was significantly reduced, but did not vanish entirely. Nitrate and protein-bound NO levels remained largely unchanged.

The finding that NO metabolite levels were not drastically reduced when animals are maintained on a low NOx diet and systemic NOS activity is inhibited was unexpected since, according to the scheme in Figure 1, these actions should have blocked all known sources of NO-related substances. To further investigate the resilience of NO products, the experiments were repeated in $\mathrm{eNOS}^{-/-}$animals subjected to dietary NOx restriction, with and without additional pharmacological inhibition of the remaining NOS isoforms. These results are depicted in Figure 4 (additional information about individual changes to S-nitrosothiol, N-nitrosamines and NOheme levels is presented in Supplementary Fig. 2). A general trend towards lower tissue protein-bound NO was observed in every organ and at the whole body level, although none sufficiently large to reach statistical significance.

Dietary restriction of nitrite and nitrate intake on top of eNOS deletion reduced plasma nitrite, whilst tissue nitrite levels remained essentially unchanged. This pattern was preserved on further NOS inhibition. Dietary depletion also had little effect on nitrate levels, with the lung being the only organ showing reduced nitrate concentrations $(p<0.05)$. Further pharmacological inhibition of remaining nNOS and iNOS in this knockout model reduced nitrate levels in the heart and kidney, in addition to a further reduction in the lung $(p<0.05)$. However, whole body levels of nitrate remained statistically unchanged (Fig. 4, lower right).

\section{Effects of combined dietary NOx restriction and chronic NOS inhibition on NO products in blood and tissues of Wistar rats}

In order to exclude the possibility of a species-specific phenomenon, additional experiments were carried out in male Wistar rats. In addition, these experiments extended the period of dietary NOx restriction and NOS inhibition and investigated the existence of a compensatory upregulation of low-molecular-weight antioxidants as a result of dietary manipulation and NOS inhibition. These animals were maintained on either regular chow/tap water, low-NOx diet/
MilliQ water, or on a regimen combining restricted dietary NOx intake and NOS inhibition. As shown in Figure 5, these studies produced qualitatively identical results, confirming our original observations in mice. Furthermore, they extend those findings to a more chronic setting. Quantitative differences in NO product concentrations between species and individual compartments notwithstanding, these data show that blood and tissue nitros(yl)ation levels overall did not drop much further after 2 weeks of dietary NOx restriction or with 1 week of NOS inhibition compared to what was observed acutely ( $3 \mathrm{~h}$ ) in mice. These effects occurred in the absence of marked changes in circulating or tissue antioxidant content (Fig. 5, right panels). Interestingly, combined dietary NOx restriction and chronic NOS inhibition revealed an intriguing additional feature that appears to be specific to the vasculature: a paradoxical upregulation of NO signaling products and metabolites in the aorta (Fig. 5), which was accompanied by a 3-fold increase in ascorbate concentration in this compartment.

\section{Discussion}

Heme nitrosylation (16) and S-nitrosation (14) represent two protein modifications that account for much of the signaling action that $\mathrm{NO}$ exerts in cells. Both types of posttranslational modifications are effected through NO or NOderived substances $(20,21)$, including nitrite (3). In all cases, the sources of these substances are associated with endogenous NO synthesis and metabolism $(2,7,8)$ or dietary NOx intake $(5,17)$. Consequently, our finding that protein-bound NO modifications persist in the absence of active NO synthesis and dietary NOx intake (e.g., in eNOS ${ }^{-/-}$animals on a low-NOx diet and under the acute action of the NOSinhibitor, L-NIO, or in Wistar rats on low-NOx diet with chronic administration of L-NMMA) is surprising since it suggests the existence of an unrecognized, and significant, source that contributes to the formation of $\mathrm{NO}$ signaling products. Our results may also explain why nitrosyl hemoglobin levels only dropped by half following dietary NOx restriction and complete NOS inhibition in another study (5). Although our results cannot pinpoint the precise nature of this new source of NO products, our results hint at the intermediacy of nitrite in the process, as we shall discuss below.

Our finding that protein-bound NO levels remain essentially unchanged upon deletion of each NOS isoform (Fig. 2, lower panels) likely reflects the action of a compensatory mechanism that maintains basal levels of $\mathrm{NO}$ metabolites in tissues. One obvious compensatory mechanism that would keep tissue NO levels constant is upregulation of remaining NOSs in an attempt to restore basal levels. If upregulation of alternative NOS isoforms alone were to take place, then there would be no physical reason why nitrite levels should change, according to the chemical network depicted in Figure 1. Yet, our results shown in Figure 2 clearly indicate that nitrite is consistently depleted, regardless of the nature of the specific NOS isoform deleted. These consistent nitrite reductions suggest that increased utilization of nitrite may be a significant component of the regulatory mechanism associated with deficient NOS expression/NOS deletion.

If inhibition of any one NOS isoform leads to increased nitrite consumption, then it must also be accompanied by increased production of nitrite elsewhere if tissues are to 

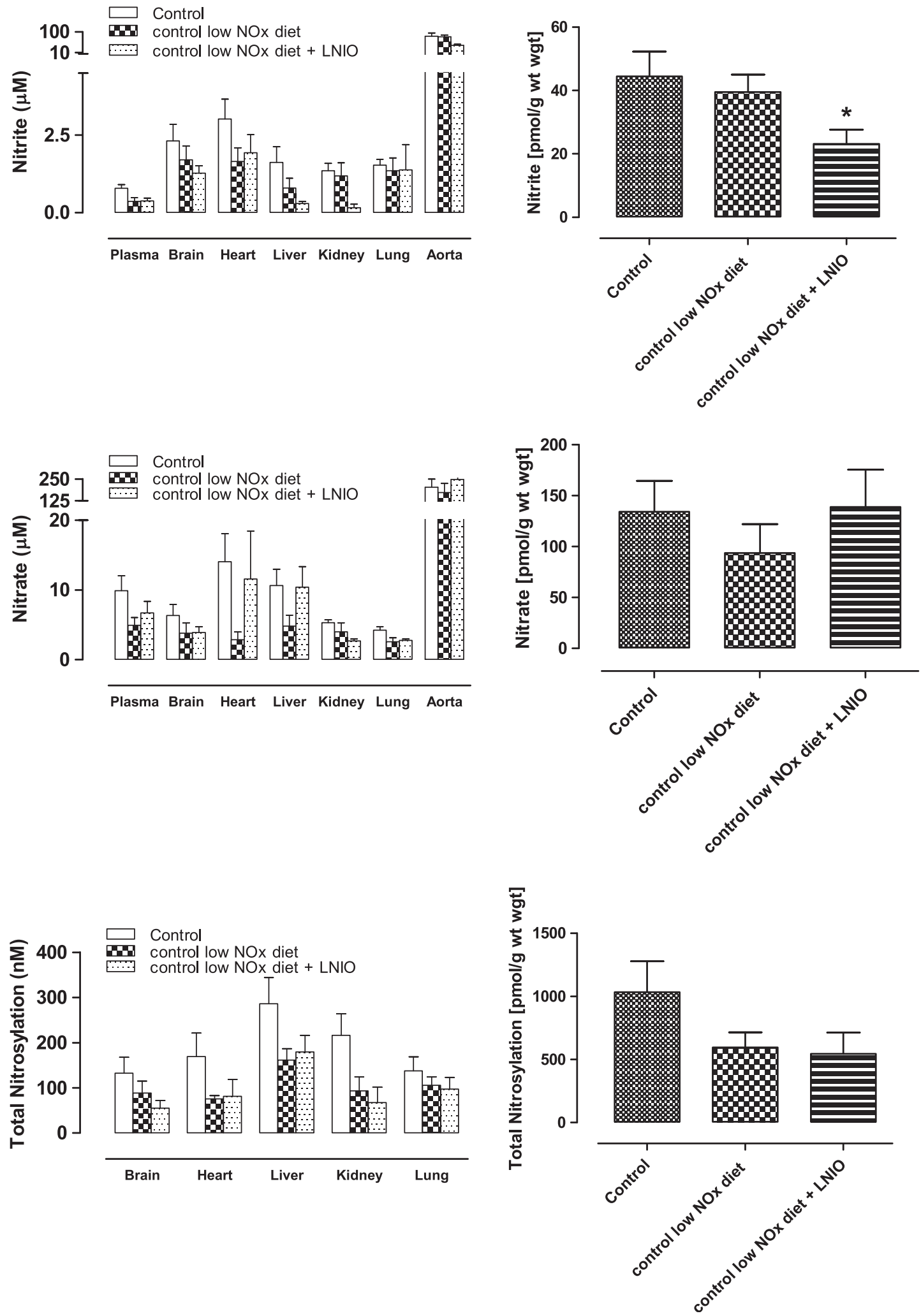

FIG. 3. Contribution of diet and other NOS isoforms to the depletion of NO metabolites in control mice. Data are from wild-type controls (C57BL/6J) $(n=8)$ kept on a standard rodent chow, and wild-type animals kept on a low NOx diet without $(n=5)$ and with additional L-NIO treatment $(n=3)$. Left-hand panels show steady-state concentrations in individual compartments, right-hand panels display a theoretical total body concentration for each NO metabolite pool expressed as a weighted average. Data are mean \pm SEM, ${ }^{*} p<0.05$. 

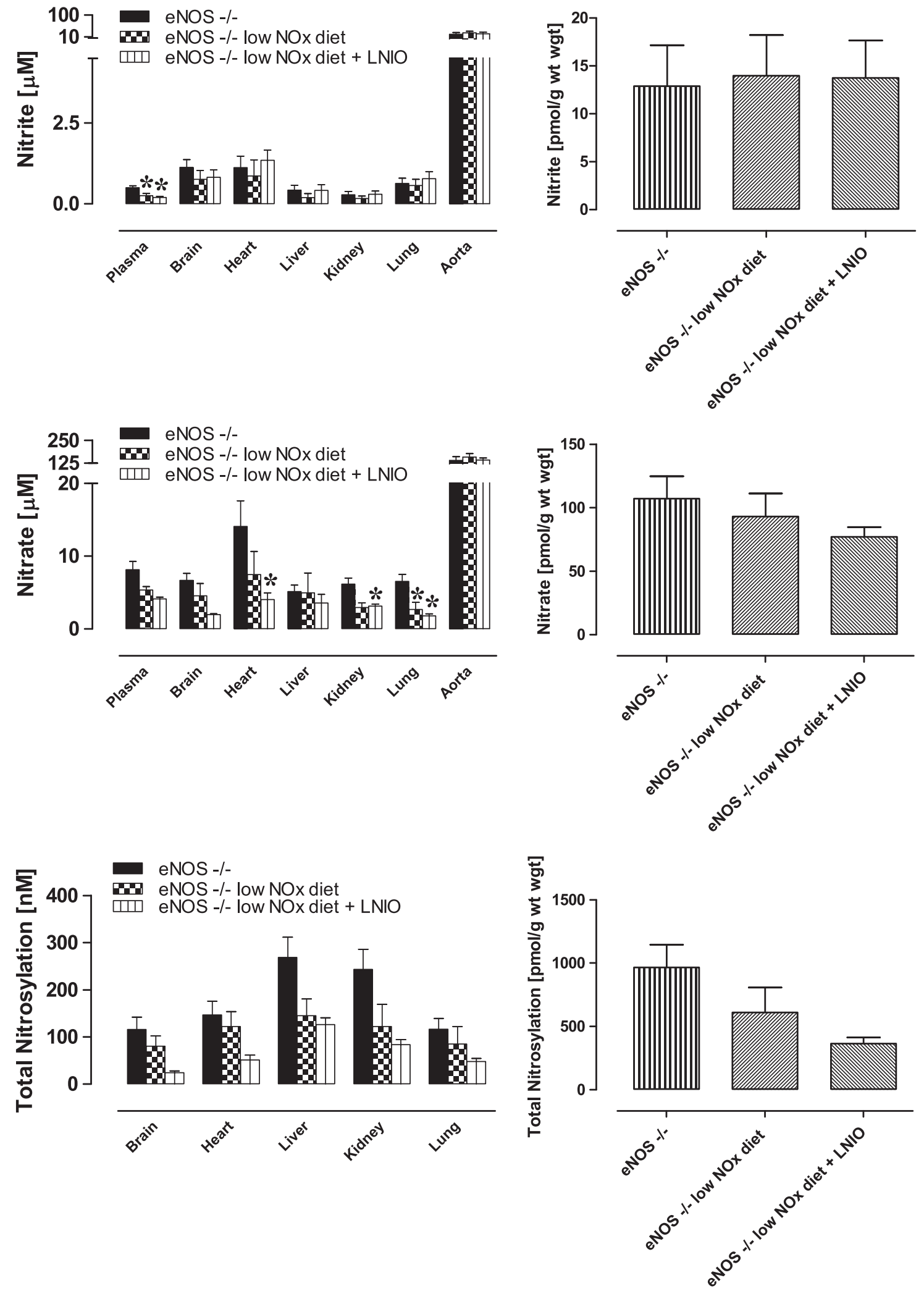

FIG. 4. Contribution of diet and other NOS isoforms to the depletion of NO metabolites in eNOS ${ }^{-l-}$ mice. Data are from eNOS $^{-/-}$animals $(n=9)$ kept on a standard rodent chow, and eNOS ${ }^{-1}$ kept on a low NOx diet without $(n=5)$ and with additional L-NIO treatment $(n=3)$. Left-hand panels show steady-state concentrations in individual compartments (individual changes in nitrosothiol, nitrosamine and NO-heme concentrations can be found in Supplementary Fig. 2 online), right-hand panels display a theoretical total body concentration for each NO metabolite pool, expressed as a weighted average. Data are mean \pm SEM, ${ }^{*} p<0.05$. 

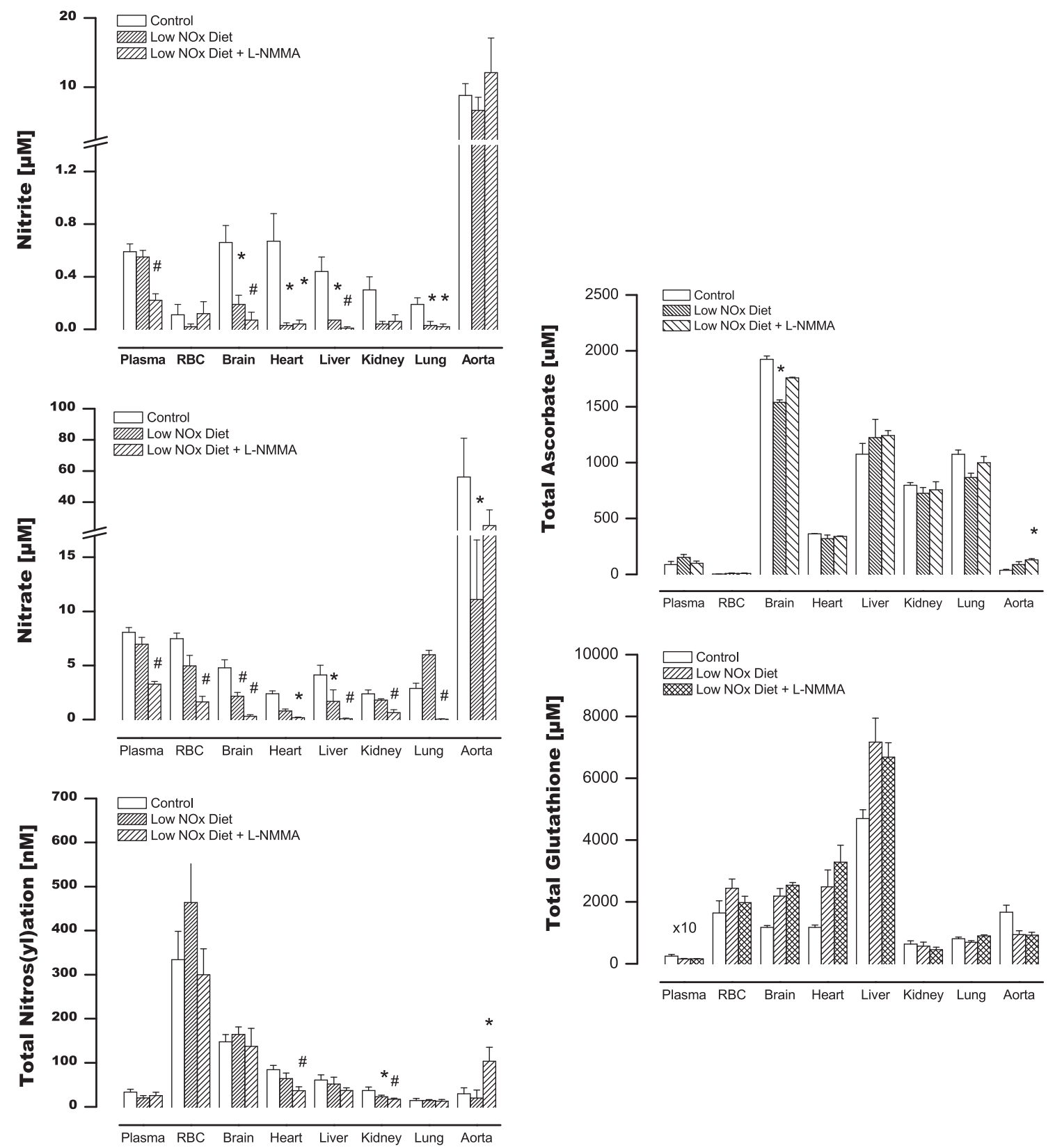

FIG. 5. Changes in NO metabolite levels upon dietary NOx restriction and chronic NOS inhibition in Wistar rats. Animals were kept either on standard rodent chow (control), low-NOx diet/MilliQ water (for 14 days), or low-NOx diet/ MilliQ water for 7 days followed by low-NOx diet/L-NMMA in MilliQ water for another week. Left-hand panels show steadystate concentrations in individual compartments, right-hand panels display a theoretical total body concentration for each NO metabolite pool expressed as a weighted average. Data are means \pm SEM of 3-5 animals/group, ${ }^{*} p<0.05$. Glutathione and ascorbate measurements were carried out in a parallel set of animals treated in an identical manner $(n=3 /$ group).

maintain a nonvanishing level of this anion. According to the nitrate-nitrite-NO pathway that has captured much attention recently $(17,18)$, such increased nitrite production could originate from the reduction of nitrate. However, the converted nitrate would also have to be replenished by some other source to sustain the pathway. For animals on a normal diet, such as those featured in Figure 2, it could be argued that the pathway may be sustained by NOx extracted from the diet. Yet our results for NOS-inhibited animals on a low-NOx diet (Figs. 3-5) reveal no evidence that effective (>98\%) dietary restriction of NOx intake leads to substantial suppression of tissue nitrite or nitrate levels. This would argue that any increased production of nitrite from nitrate would ultimately have to be derived from some source not considered in our scheme depicted in Figure 1.

The notion of NOS-independent NO production in mammalian tissues is not new (37). However, the effects we here observe using NOS knockout animals are larger than usually assumed. Since rodents synthesize ascorbic acid, and ascorbate has been shown to promote NO generation from nitrite reduction, either by direct chemical reaction (28) or by xanthine oxidase-catalyzed reduction in tissues (18), adaptive 


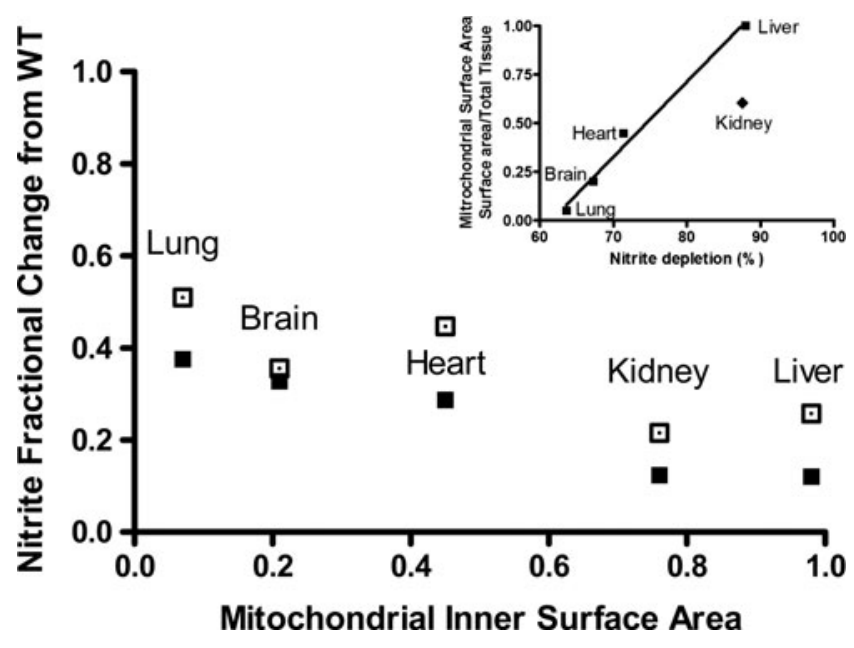

FIG. 6. Fractional change in steady-state concentrations of nitrite in organs from eNOS ${ }^{-1-}$ mice maintained on a lowNOx diet, with and without additional NOS inhibition. All values are expressed relative to those found in the same organs of wild-type (WT) animals fed a standard rodent chow, and plotted against total tissue mitochondrial inner surface area (data from Reference 15) for each organ; closed symbols: $\mathrm{eNOS}^{-/-}$on low NOX diet; open symbols: $\mathrm{NOS}^{-/-}$on low NOX diet plus L-NIO. Inset: Correlation between mitochondrial inner surface area and nitrite depletion, expressed as percent of control levels in wild-type mice, following removal of all currently known endogenous and exogenous supplies of nitrite $\left(r^{2}=0.989\right.$, excluding the kidney).

changes in ascorbate levels may have taken place to attenuate the drop of NOS-dependent NO production. This possibility was investigated in rats subjected to chronic NOS inhibition rather than by comparing wild-type with $\mathrm{NOS}^{-/-}$mice. In most compartments, changes in ascorbate content in response to restriction of NOx intake and chronic NOS inhibition were moderate. Only in the aorta, ascorbate increased significantly while glutathione levels dropped, and even these changes are not accompanied by nitrite depletion. Thus, the data does not support the notion that tissue nitrite reduction is enhanced due to increased ascorbate content and/or recycling. Moreover, administration of the xanthine oxidase inhibitor allopurinol did not alter nitrite levels significantly (not shown). Taken together, these results suggest that neither ascorbate nor xanthine oxidase make a major contribution to total $\mathrm{NO}$ product formation in vivo.

Whether nitrite plays a central role in sustaining NO signaling in the absence of NOS activity, an inescapable conclusion from our results is that NO-related substances continue to be synthesized in mice even after dietary NOx is highly reduced and NOS is inhibited. Our study in mice is limited inasmuch as we did not extend the period of NOS inhibition beyond 3 hours (in order to avoid stress as a possible confounder). Nevertheless, this period should have been more than enough to see substantial changes in nitrite and protein-bound NO levels as it corresponds to twice the plasma half-life of nitrate in mice (34), the NO metabolite with the longest half-life in vivo and the largest pool of NO products in the body. In the absence of other sources of intake or production, whole-body nitrate levels should have dropped by $75 \%$ within that time if NOS was completely inhibited. Yet, such level of reduction is not observed. Considering that reversible enzyme inhibitors were used, one could argue that NOS inhibition may have been incomplete. However, our more chronic NOS inhibitor studies in rats yielded similar results. Had the degree of enzyme inhibition in the acute studies in mice and in the chronic studies in rats not both been close to maximal, such similarity in outcome would have been highly unlikely.

An intriguing additional feature emerging from the rat studies was the paradoxical upregulation of NO products during chronic combined dietary NOx restriction and pharmacological NOS inhibition in aortic tissue. The observed increase of NO products in the aorta following NOS inhibition may be secondary to enhanced NOx uptake from the circulation, indicative of an enhanced need for such products in the vasculature when NO production from L-arginine is compromised.

However surprising these findings may be, they can only be interpreted as evidence that a source other than those depicted in Figure 1 may be sustaining the nitros(yl)ation/NOx cycle at basal levels when NOS is inhibited. This source, although unidentified at this time, may be a key to understanding why even triple NOS knockout mice remain viable.

At this stage, we can only speculate about the origin of this elusive source of NO products. Future studies under specific pathogen-free conditions may provide insight as to whether it originates from microbial or mammalian activities. Further investigations, requiring the use of tracer techniques such as ${ }^{15} \mathrm{~N}$-labeling in combination with isotope-specific analytical methods, may reveal that the unknown pool of NO metabolites is derived from the oxidation of ammonia, metabolism of hydroxylamine, uptake/metabolism of a nitrogen species in air, or perhaps "recycling" of urea. Other possibilities may include reductive biotransformation of nitrated species. Alternatively, it may be a by-product of protein catabolism/ turnover and derived from the $\alpha$-amino group of amino acids.

Finally, we wish to point out an interesting observation that may bear on the role of nitrite in NOx-restricted, NOS-deleted animals. As stated earlier, these animals exhibit significantly reduced levels of whole-body nitrite compared to those of WT animals. However, when one examines the concentration changes relative to WT for each organ, the level of reduction varies widely from one organ to another (Fig. 6). In fact, the level of reduction appears to vary systematically with tissue mitochondrial inner surface area (a proxy for maximal oxidative phosphorylative capacity of that tissue; data taken from 15) as suggested in that figure. This observation confirms and extends earlier in vitro findings (10) related to the nitrite reductase activity of the same tissues to the in vivo situation. This link suggests that the nitrite depletion we observe in tissues may be related to mitochondrial activity and perhaps it holds a key to unlocking the mystery of how basal NO metabolite concentrations (and, presumably, related downstream signaling) is sustained in the absence of NOS and dietary NOx pathways.

\section{Materials and Methods}

\section{Materials}

All chemicals were of the highest purity available and purchased from Sigma-Aldrich (St. Louis, MO) unless otherwise specified. 


\section{Animals}

Ten-week-old male mice of the $\mathrm{iNOS}^{-/-}$and $\mathrm{eNOS}^{-/-}$ strains (Jackson Laboratory, Ben Harbor, ME; stock \# 002684 and 002609) were compared with age-matched C57BL/6J wild-type mice (WT; Jackson Laboratory), and $\mathrm{nNOS}^{-/-}$ mice (Jackson; \#002633) were compared with their corresponding genetic background strain, B6129SF2/J (Jackson; \#101045). No significant differences in basal levels of NO metabolites were observed between the two WT strains (data not shown). Male Wistar rats (10-12 weeks of age) were purchased from Harlan (South Easton, MA). Animals were maintained on a standard rodent chow (2018; Harlan Teklad, South Easton, MA), with food and tap water ad libitum, and kept on a normal 12/12h light cycle. A minimum of 10 days was allowed for local vivarium acclimatization prior to experimental use. All experiments complied with federal and state regulations in accordance with the Guide for the Care and Use of Laboratory Animals (Institute for Laboratory Animal Research, National Research Council) and were approved by the Institutional Animal Care and Use Committee of the Boston University School of Medicine.

\section{Dietary depletion of nitrite and nitrate}

To deplete animals of their dietary source of nitrite and nitrate, a subset of acclimatized animals was switched to receive an amino acid diet (TD99366; Harlan Teklad) with matched L-arginine content $(1.2 \%$ vs. $1 \%$ in the standard rodent diet) and MilliQ water for 1 week prior to experimental analysis. This particular diet has considerably lower levels of nitrite/nitrate $\left(1.5 / 16.3 \mathrm{nmol} \mathrm{g}^{-1}\right)$ when compared with the standard chow $\left(5.4 / 118 \mathrm{nmol} \mathrm{g}^{-1}\right)$ (3). Drinking water contributes significantly to total daily intake as NOx concentrations approach maximal contaminant levels of nitrite/nitrate. Based on strain-specific information about food and water consumption (1) and measured NOx content of the water provided the daily intake of food and water-derived nitrite/nitrate on the standard diet/tap water regimen was calculated to be $0.46 / 4.81 \mu$ moles nitrite/ nitrate compared with the distilled water/amino acid diet (referred to hereafter as the "low-NOx" diet) of 6/67 nmoles for a $25 \mathrm{~g}$ mouse, corresponding to a $>98.5 \%$ reduction in total NOx intake on the latter compared to the regular diet regimen.

\section{NOS inhibitor treatment}

To investigate possible contributions from other NOS isoforms the pan-NOS inhibitor, L-N-iminoethyl-ornithine (L-NIO hydrochloride; A.G. Scientific, Inc., San Diego, CA) was administered for $3 \mathrm{~h}$ using a dose regimen confirmed to ensure maximal NOS inhibition $(100 \mathrm{mg} / \mathrm{kg}$ s.c., every $45 \mathrm{~min}$ ) (2) prior to measurement in both wt and $\mathrm{eNOS}^{-/-}$ animals fed a low NOx diet. In separate experiments in rats, L-N-monomethyl-arginine (L-NMMA acetate, $1 \mathrm{mg} / \mathrm{mL}$ dissolved in MilliQ water; 11) was administered for 7 days with the drinking water while animals were kept on a lowNOx diet, with controls receiving either normal rodent chow/tap water or low-NOx diet/MilliQ water. We opted to employ L-NMMA instead of L-N ${ }^{\mathrm{G}}$-nitroarginine methylester (L-NAME, $0.3 \mathrm{mg} / \mathrm{mL}$; 25) as NOS inhibitor for two reasons: i) L-NAME, but not L-NMMA, exerts NOS- independent effects by virtue of muscarinic receptor antagonistic activity (4); ii) earlier pilot experiments with this compound revealed that it is metabolized to nitrite and nitrate (particularly in the liver from which these anions appear to leak into the circulation), confounding the analysis of NO metabolite status.

\section{Organ harvest and tissue homogenization}

Heparinized (0.07 units/g body weight, i.p.) mice or rats were anaesthetized with diethylether $(2 \mathrm{~min})$ and euthanized by cervical dislocation. Blood was collected by cardiac puncture, and plasma and red blood cells were obtained by centrifugation. After thoracotomy, a catheter was inserted into the infrarenal part of the abdominal aorta, and organs were flushed free of blood by retrograde in situ perfusion with air-equilibrated phosphate-buffered saline supplemented with N-ethylmaleimide/ethylenediamine tetraacetic acid $(10 / 2.5 \mathrm{mM})$ at a rate of $10 \mathrm{ml} / \mathrm{min}$, and organs were harvested as detailed previously (2). To provide sufficient sample for comprehensive analysis of $\mathrm{NO}$ metabolite levels in animals lacking individual NOS isoform, samples were pooled from three animals; in all subsequent experiments on $\mathrm{eNOS}^{-/-}$groups, analysis was performed on individual tissue and blood samples. No significant differences in levels were observed between pooled and individual measurements. Despite pooling, volumes of murine plasma and aorta samples were insufficient for an accurate determination of nitrosation levels, allowing us to only analyze nitrite and nitrate concentrations in these compartments.

\section{Determination of tissue nitroso/nitrosyl, nitrite/nitrate, ascorbate, and glutathione content}

Tissue levels of nitroso and nitrosyl compounds were quantified using group-specific reductive denitrosation by iodine-iodide with subsequent detection of liberated NO by gas-phase chemiluminescence (9). "RSNO" signifies mercurylabile S-nitroso species, whereas "RNNO" signifies mercuryresistant $\mathrm{N}$-nitroso adducts and may include nitrosamines and metal nitrosyls other than NO-heme species. NO-heme, a product of heme nitrosylation, was determined by injection of replicate aliquots of tissue homogenates into a solution of 0.05 $M$ ferricyanide in PBS at $\mathrm{pH} 7.5$ and $37^{\circ} \mathrm{C}$ (2). This method employs one-electron oxidation rather than reduction to achieve denitrosation and NO release. Nitrate and nitrite in plasma and tissues were quantified by ion chromatography with on-line reduction of nitrate to nitrite and post-column Griess diazotization (ENO-20 Analyzer; Eicom, Kyoto, Japan) (2). Total ascorbate and glutathione concentrations in blood and tissues were analyzed as described previously (2). In some animals, allopurinol $(100 \mathrm{mg} / \mathrm{kg}$ ip $)$ was administered $30 \mathrm{~min}$ prior to organ harvest.

\section{Statistical analysis}

Data are means \pm SEM from $n$ individual experiments. Statistical analysis was performed using Graph Pad Prism 4.0. Comparison between groups was achieved by one-way ANOVA following Bonferroni correction, or Dunnett's posthoc test where appropriate. Statistical significance was determined by $p<0.05$. 


\section{Acknowledgments}

We wish to thank S.M. Bauer and N. S. Bryan for skillful technical assistance during the initial parts of the study. This work was supported by grants from the National Institute of Health (HL 69029 and DA020644 to M.F., P20 RR16456 from the BRIN/INBRE Program of the NCRR to JR) and the Strategic Appointment Scheme of the Medical Research Council, UK (to MF).

\section{Author Disclosure Statement} thors.

No competing financial interests exist for any of the au-

\section{References}

1. Bachmanov AA, Reed DR, Beauchamp GK, and Tordoff MG. Food intake, water intake, and drinking spout side preference of 28 mouse strains. Behav Genet 32: 435-443, 2002.

2. Bryan NS, Rassaf T, Maloney RE, Rodriguez CM, Saijo F, Rodriguez JR, and Feelisch M. Cellular targets and mechanisms of nitros(yl)ation: An insight into their nature and kinetics in vivo. Proc Natl Acad Sci USA 101: 4308-4313, 2004.

3. Bryan NS, Fernandez BO, Bauer SM, Garcia-Saura MF, Milsom AB, Rassaf T, Maloney RE, Bharti A, Rodriguez J, and Feelisch M. Nitrite is a signaling molecule and regulator of gene expression in mammalian tissues. Nat Chem Biol 1: 290-297, 2005.

4. Buxton IL, Cheek DJ, Eckman D, Westfall DP, Sanders KM, and Keef KD. $\mathrm{N}^{\mathrm{G}}$-nitro L-arginine methyl ester and other alkyl esters of arginine are muscarinic receptor antagonists. Circ Res. 72: 387-395, 1993.

5. Dikalov S and Fink B. ESR techniques for the detection of nitric oxide in vivo and in tissues. Methods Enzymol 396: 597610, 2005.

6. Donzelli S, Switzer CH, Thomas DD, Ridnour LA, Espey MG, Isenberg JS, Tocchetti CG, King SB, Lazzarino G, Miranda KM, Roberts DD, Feelisch M, and Wink DA. The activation of metabolites of nitric oxide synthase by metals is both redox and oxygen dependent: A new feature of nitrogen oxide signaling. Antioxid Redox Signal 8: 1363-1371, 2006.

7. Dyson A, Bryan NS, Fernandez BO, Garcia-Saura MF, Saijo F, Mongardon N, Rodriguez J, Singer M, and Feelisch M. An integrated approach to assessing nitroso-redox balance in systemic inflammation. Free Radic Biol Med 51: 1137-1145, 2011.

8. Elrod JW, Greer JJ, Bryan NS, Langston W, Szot JF, Gebregzlabher H, Janssens S, Feelisch M, and Lefer DJ. Cardiomyocyte-specific overexpression of $\mathrm{NO}$ synthase- 3 protects against myocardial ischemia-reperfusion injury. Arterioscler Thromb Vasc Biol 26: 1517-1523, 2006.

9. Feelisch M, Rassaf T, Mnaimneh S, Singh N, Bryan NS, Jourd'Heuil D, and Kelm M. Concomitant S-, N-, and hemenitros(yl)ation in biological tissues and fluids: Implications for the fate of NO in vivo. FASEB J 16: 1775-1785, 2002.

10. Feelisch M, Fernandez BO, Bryan NS, Garcia-Saura MF, Bauer S, Whitlock DR, Ford PC, Janero DR, Rodriguez J, and Ashrafian $\mathrm{H}$. Tissue processing of nitrite in hypoxia: An intricate interplay of nitric oxide-generating and -scavenging systems. J Biol Chem 283: 33927-33934, 2008.

11. Gardiner SM, Kemp PA, and Bennett T. Effects of chronic treatment with nitric oxide synthase inhibitors on regional haemodynamic responses to vasodilators in conscious Brattleboro rats. Br J Pharmacol 109: 222-228, 1993.

12. Gödecke A and Schrader J. Adaptive mechanisms of the cardiovascular system in transgenic mice-Lessons from eNOS and myoglobin knockout mice. Basic Res Cardiol 95: 492-498, 2000.

13. Gross SS and Wolin MS. Nitric oxide: Pathophysiological mechanisms. Annu Rev Physiol 57: 737-769, 1995.

14. Hess DT, Matsumoto A, Kim SO, Marshall HE, and Stamler JS. Protein S-nitrosylation: Purview and parameters. Nat Rev Mol Cell Biol 6: 150-166, 2005.

15. Hulbert AJ and Else PL. Evolution of mammalian endothermic metabolism: Mitochondrial activity and cell composition. Am J Physiol 256: R63-R69, 1989.

16. Ignarro LJ. Signal transduction mechanisms involving nitric oxide. Biochem Pharmacol 41: 485-490, 1991.

17. Lundberg JO, Weitzberg E, and Gladwin MT. The nitratenitrite-nitric oxide pathway in physiology and therapeutics. Nat Rev Drug Discov 7: 156-167, 2008.

18. Lundberg JO, Gladwin MT, Ahluwalia A, Benjamin N, Bryan NS, Butler A, Cabrales P, Fago A, Feelisch M, Ford PC, Freeman BA, Frenneaux M, Friedman J, Kelm M, Kevil CG, Kim-Shapiro DB, Kozlov AV, Lancaster JR Jr, Lefer DJ, McColl K, McCurry K, Patel RP, Petersson J, Rassaf T, Reutov VP, Richter-Addo GB, Schechter A, Shiva S, Tsuchiya K, van Faassen EE, Webb AJ, Zuckerbraun BS, Zweier JL, and Weitzberg E. Nitrate and nitrite in biology, nutrition and therapeutics. Nat Chem Biol 5: 865-869, 2009.

19. Mergia E, Koesling D, and Friebe A. Genetic mouse models of the NO receptor 'soluble' guanylyl cyclases. Handb Exp Pharmacol 191: 33-46, 2009.

20. Miersch S and Mutus B. Protein S-nitrosation: Biochemistry and characterization of protein thiol-NO interactions as cellular signals. Clin Biochem 38: 777-791, 2005.

21. Miranda KM, Nims RW, Thomas DD, Espey MG, Citrin D, Bartberger MD, Paolocci N, Fukuto JM, Feelisch M, and Wink DA. Comparison of the reactivity of nitric oxide and nitroxyl with heme proteins. A chemical discussion of the differential biological effects of these redox related products of NOS. J Inorg Biochem 93: 52-60, 2003.

22. Moncada S, Palmer RM, and Higgs EA. Nitric oxide: Physiology, pathophysiology, and pharmacology. Pharmacol Rev 43: 109-142, 1991.

23. Nagasaka Y, Fernandez BO, Garcia-Saura MF, Petersen B, Ichinose F, Bloch KD, Feelisch M, and Zapol WM. Brief periods of nitric oxide inhalation protect against myocardial ischemia-reperfusion injury. Anesthesiology 109: 675-682, 2008.

24. Nathan C and Xie QW. Nitric oxide synthases: Roles, tolls, and controls. Cell 78: 915-918, 1994.

25. Navarro J, Sanchez A, Sáiz J, Ruilope LM, García-Estań J, Romero JC, Moncada S, and Lahera V. Hormonal, renal, and metabolic alterations during hypertension induced by chronic inhibition of NO in rats. Am J Physiol 267: R1516R1521, 1994.

26. Padgett $\mathrm{CM}$ and Whorton AR. Regulation of cellular thiol redox status by nitric oxide. Cell Biochem Biophys 27: 157-177, 1995.

27. Rassaf T, Feelisch M, and Kelm M. Circulating NO pool: Assessment of nitrite and nitroso species in blood and tissues. Free Radic Biol Med 36: 413-422, 2004.

28. Rocha BS, Gago B, Barbosa RM, and Laranjinha J. Diffusion of nitric oxide through the gastric wall upon reduction of 
nitrite by red wine: Physiological impact. Nitric Oxide 22: 235-241, 2010.

29. Rodriguez J, Maloney RE, Rassaf T, Bryan NS, and Feelisch $\mathrm{M}$. Chemical nature of nitric oxide storage forms in rat vascular tissue. Proc Natl Acad Sci USA 100: 336-341, 2003.

30. Shimokawa $H$ and Tsutsui $M$. Nitric oxide synthases in the pathogenesis of cardiovascular disease: Lessons from genetically modified mice. Pflugers Arch 459: 959-967, 2010.

31. Stamler JS, Lamas S, and Fang FC. Nitrosylation. The prototypic redox-based signaling mechanism. Cell 106: 675-683, 2001.

32. Thomas DD, Ridnour LA, Isenberg JS, Flores-Santana W, Switzer CH, Donzelli S, Hussain P, Vecoli C, Paolocci N, Ambs S, Colton CA, Harris CC, Roberts DD, and Wink DA. The chemical biology of nitric oxide: Implications in cellular signaling. Free Radic Biol Med 45: 18-31, 2008.

33. Tsutsui M, Shimokawa H, Morishita T, Nakashima $Y$, and Yanagihara N. Development of genetically engineered mice lacking all three nitric oxide synthases. J Pharmacol Sci 102: 147-154, 2006.

34. Veszelovsky E, Holford NH, Thomsen LL, Knowles RG, and Baguley BC. Plasma nitrate clearance in mice: Modeling of the systemic production of nitrate following the induction of nitric oxide synthesis. Cancer Chemother Pharmacol 36: 155159, 1995.

35. Walford G and Loscalzo J. Nitric oxide in vascular biology. $J$ Thromb Haemost 1: 2112-2118, 2003.

36. Wink DA, Miranda KM, Espey MG, Pluta RM, Hewett SJ, Colton C, Vitek M, Feelisch M, and Grisham MB. Mechanisms of the antioxidant effects of nitric oxide. Antioxid Redox Signal 3: 203-213, 2001.
37. Zweier JL, Samouilov A, and Kuppusamy P. Non-enzymatic nitric oxide synthesis in biological systems. Biochim Biophys Acta 1411: 250-262, 1999.

Address correspondence to: Prof. Martin Feelisch Experimental Medicine and Integrative Biology University of Warwick Medical School Gibbet Hill Road Coventry CV4 7AL United Kingdom

E-mail:mf@warwick.ac.uk

Date of first submission to ARS Central, July 12, 2011; date of final revised submission, December 1, 2011; date of acceptance, December 1, 2011.

\begin{tabular}{|c|} 
Abbreviations Used \\
eNOS $=$ endothelial nitric oxide synthase \\
$\mathrm{iNOS}=$ inducible nitric oxide synthase \\
$\mathrm{L}-\mathrm{NIO}=\mathrm{L}-\mathrm{N}-$-iminoethyl-ornithine \\
$\mathrm{L}-\mathrm{NMM}=\mathrm{L}-\mathrm{N}-$ monomethyl-arginine \\
$\mathrm{nNOS}=$ neuronal nitric oxide synthase \\
$\mathrm{NO}=$ nitric oxide \\
$\mathrm{NOS}=$ nitric oxide synthase \\
$\mathrm{NOx}=$ sum of nitrite and nitrate \\
$\mathrm{WT}=$ wild-type
\end{tabular}

